\title{
Study on Improvement of MPPT Efficiency in PV Generation System with Partial Shadow
}

\author{
Kazutaka Itako and Masataka Ochiai \\ Electrical \& Electronics Engineering Department, Kanagawa Institute of Technology, 1030 Shimo-Ogino, Atsugi 243-0292, Japan
}

Received: October 09, 2017 / Accepted: October 19, 2017 / Published: November 30, 2017

\begin{abstract}
The maximum power point of PV (photovoltaic) generation moves depending on weather conditions and load. Therefore, it is significant to make sure that the panels can work at the maximum power point under MPPT (maximum power point tracking) control. However, it has the problems of low efficiency and unstable operation when panels are covered by the partial shadow. The result is that the output power may be substantially decreased. To overcome this issue, the authors propose a new plug-in operation point correction system. This system is put between PV panels and PCS (power conditioning system) in the existing PV generation system. In this paper, the experimental results describe that the output electric energy increases approximately 1.4 times as compared with the conventional system when the proposed correction system is inserted.
\end{abstract}

Key words: PV generation system, operation point correction, MPPT, partial shadow.

\section{Introduction}

The maximum power point of PV generation moves depending on weather conditions and load. Therefore, it is significant to make sure that the panels can work at the maximum power point under MPPT (maximum power point tracking) control [1-4].

However, it has the problems of low efficiency and unstable operation when panels are covered by the partial shadow. The result is that the output power may be substantially decreased. In order to solve this problem, the authors proposed new algorithm which detects maximum power point, scanning the I-V characteristics [5-10]. As this method is mounted into the PCS (power conditioning system), it is suitable for new installation of PV generation system.

This paper proposes a new plug-in operation point correction system which corrects the operating point of PV panels in the existing PV generation system [11-13].

Corresponding author: Kazutaka Itako, Ph.D., professor, research fields: power electronics, renewable energy.

\section{Outline of Conventional PV Generation System}

Fig. 1 shows the configuration diagram of the conventional PV generation system. The output voltage of PV panels is applied to the PCS input. The applied DC voltage is converted to the AC voltage as an output voltage of the PCS. The PCS output is connected to the power grid. The PCS has two main functions. One is "MPPT function", the other one is "inverter function". In the PCS, the P\&O method is normally used as MPPT algorithm.

Fig. 2 shows the P-V characteristic (without shadow $\&$ with shadow). In the PV generation system with a shadow, because of the function of the bypass diode, two or more peaks occur in the P-V characteristic, as shown in the figure. The $\mathrm{P} \& \mathrm{O}$ method may operate at a lower peak point voltage when there are two peaks in the P-V characteristic as shown in Fig. 2. This is a problem of the conventional MPPT method.

\section{Proposed PV Generation System}

The system configuration diagram of proposed system is shown in Fig. 3. By inserting an operation 


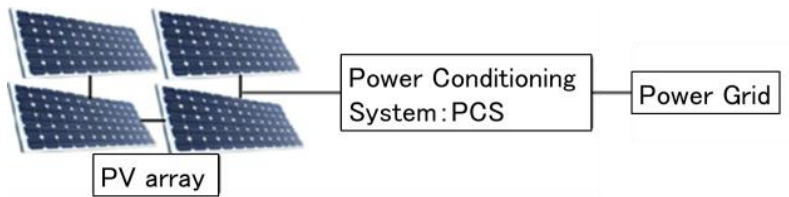

Fig. 1 Configuration diagram of conventional PV system.

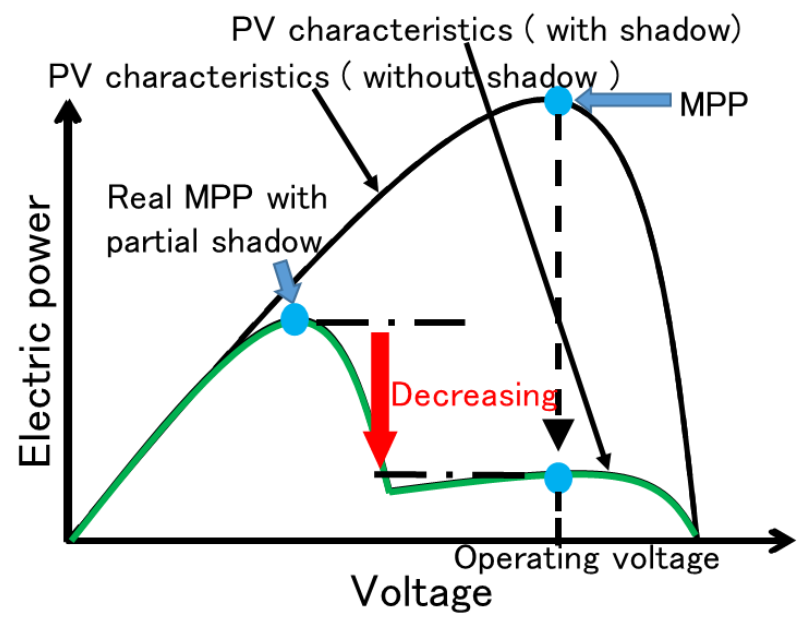

Fig. 2 P-V characteristic (without shadow \& with shadow).

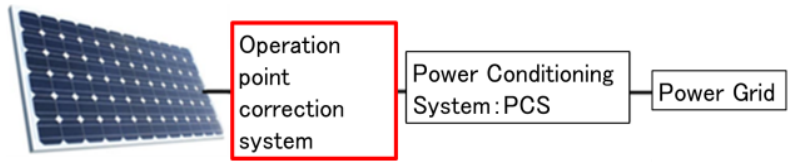

Fig. 3 Configuration diagram of new plug-in operation point correction system.

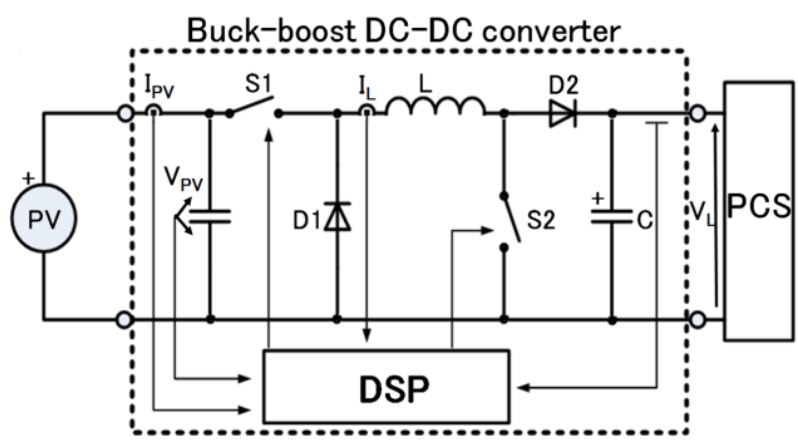

Fig. 4 System configuration.

point correction system between panel and PCS, it becomes possible that the PV generation system covered by partial shadows can operate at the maximum power point through the P\&O method of the PCS. Fig. 4 shows the configuration diagram of the developed operation point correction system. This developed system is the section enclosed in the dotted line and it consists of a buck-boost type DC-DC converter.

The operating voltage of the PCS has an operable range. It is necessary to distinguish the operation depending on whether $V_{\mathrm{OP}}$ at the maximum power point is within the PCS operable range (pattern A) or out of the range (pattern $B$ ).

\subsection{Concept of Operation at Pattern A}

$V_{\text {Lmin }}$ is the lowest value of range of the PCS input operating voltage. Pattern $\mathrm{A}$ is in the case that $V_{\mathrm{OP}}$ is $V_{\text {Lmin }}$ or more. Fig. 5 shows a conceptual diagram of operation in the case of pattern $\mathrm{A}$, where there are two peaks in the $\mathrm{P}-\mathrm{V}$ characteristic. This system generates the output power along with the arrow (red dotted line) and directs the operating point voltage $V_{\mathrm{L}}$ to the maximum power point voltage $V_{\mathrm{OP}}$ (bridge operation). In other words, this system only supports the MPPT of the PCS.

\subsection{Concept of Operation at Pattern B}

Fig. 6 shows the operation conceptual diagram of pattern $\mathrm{B}\left(V_{\mathrm{OP}}\right.$ is less than $\left.V_{\mathrm{L}}\right)$. In this case, the maximum power point voltage $V_{\mathrm{OP}}$ is out of the range of the PCS input operating voltage. The maximum power point $P_{\mathrm{MAX}}$ can be moved into the operable range by using switching control with a DC-DC converter, which performs boost operation with a constant step-up ratio $\mathrm{n}$. The step-up ratio $\mathrm{n}$ is,

$$
n=V_{\mathrm{L}} / V_{\mathrm{OP}}
$$

Proposed system in pattern B always boosts the operating point voltage, and proposed system performs MPPT with P\&O method of the PCS.

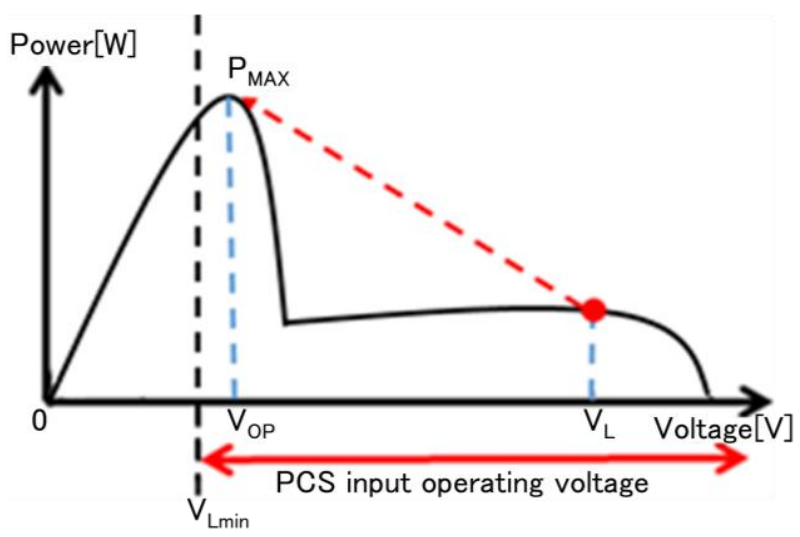

Fig. 5 Conceptual diagram of pattern A. 


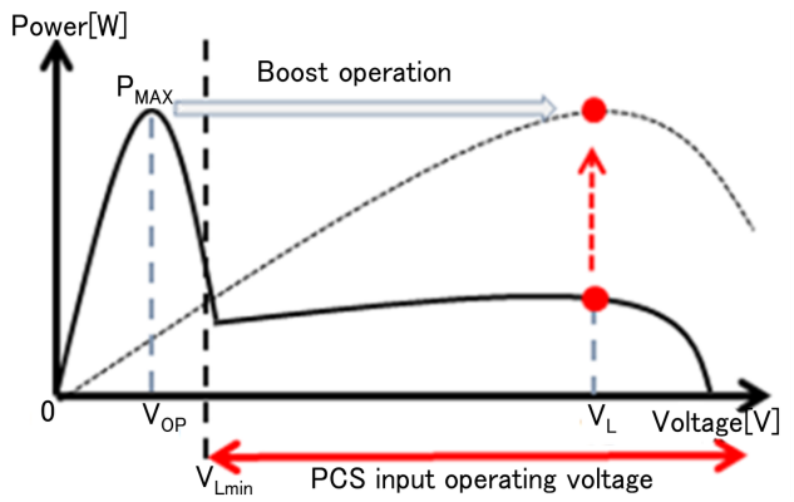

Fig. 6 Conceptual diagram of pattern $B$.

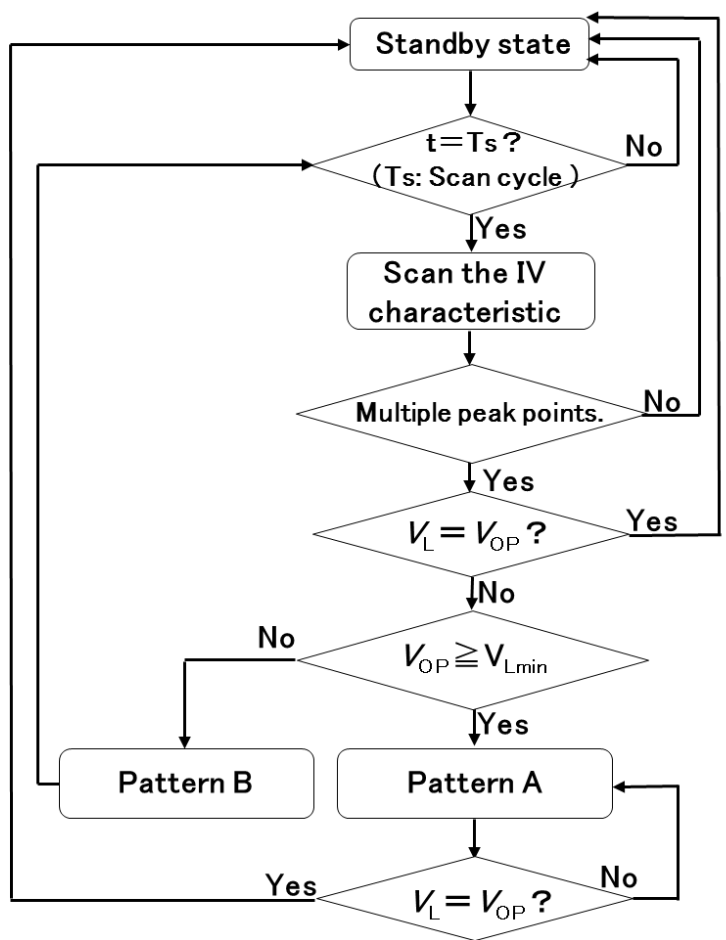

Fig. 7 Flowchart of the proposed system.

Fig. 7 shows the flowchart of the proposed system. In the standby mode, the output of PV panels is sent directly to the PCS. Next, when $t=T_{\mathrm{S}}$ (scan cycle), the proposed system scans the $I-V$ characteristics to detect $V_{\mathrm{OP}}$ and makes a comparison with $V_{\mathrm{L}}$. And, the system confirms whether the multiple peak points exist. When $V_{\mathrm{L}}$ is equal to $V_{\mathrm{OP}}$, the correction system returns to standby mode. If $V_{\mathrm{L}}$ is not equal to $V_{\mathrm{OP}}$, the system compares $V_{\mathrm{OP}}$ and $V_{\mathrm{Lmin}}$.

When $V_{\mathrm{OP}}$ is $V_{\mathrm{L}}$ or more, the proposed system operates on pattern A. When $V_{\mathrm{L}}$ equals to $V_{\mathrm{OP}}$, the correction system returns to standby mode. When $V_{\mathrm{OP}}$ is less than $V_{\mathrm{L}}$, the proposed system operates on pattern B. Using the step-up ratio $\mathrm{n}$ calculated by Eq. (1), the converter achieves the step-up operation.

\section{Experimental Results}

\subsection{Operation Confirmation Experiment}

Fig. 8 shows the experimental circuit. In this experiment, PV simulator is used instead of PV panels, and $\mathrm{P} \& \mathrm{O}$ unit is used instead of PCS.

Table 1 shows the specifications of PV simulator. $I-V$ characteristics are able to change for solar radiation intensity. Operation point correction system is placed between $\mathrm{PV}$ simulator and $\mathrm{P} \& \mathrm{O}$ unit.

Fig. 9 shows the $P-V$ characteristics of the PV simulator considering the partial shadow. The $V_{\mathrm{OP}}$ of the PV simulator is about $15 \mathrm{~V}$.

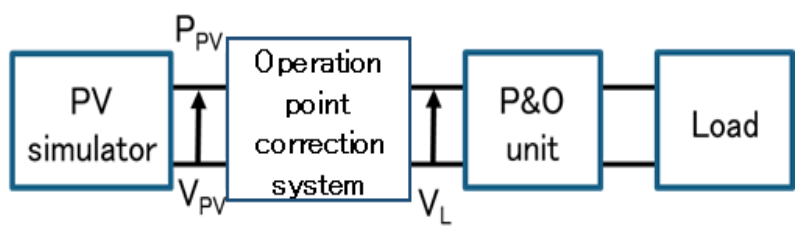

Fig. 8 Experimental circuit.

Table 1 Specifications of PV simulator.

\begin{tabular}{ll}
\hline Maximum output $P_{\mathrm{MAX}}$ & $91.6 \mathrm{~W}$ \\
Open circuit voltage $V_{\mathrm{OC}}$ & $38.7 \mathrm{~V}$ \\
Short circuit current $I_{\mathrm{SC}}$ & $3.67 \mathrm{~A}$ \\
Voltage at maximum output $V_{\mathrm{OP}}$ & $28.2 \mathrm{~V}$ \\
Current at maximum output $I_{\mathrm{OP}}$ & $3.25 \mathrm{~A}$ \\
\hline
\end{tabular}

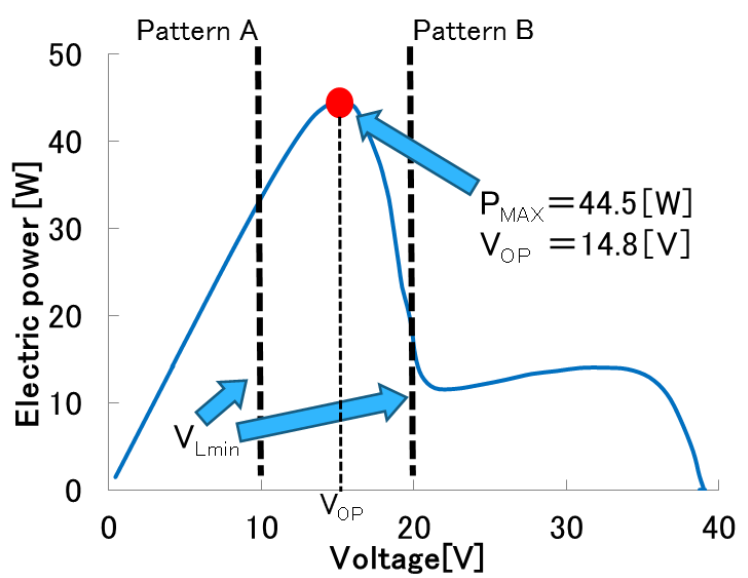

Fig. $9 P-V$ characteristic of $P V$ simulator considering the partial shadow. 
In this $P-V$ characteristic, if $V_{\mathrm{Lmin}}$ is set to $10 \mathrm{~V}$ then the system condition is in pattern $\mathrm{A}$. And if $V_{\mathrm{Lmin}}$ is set to $20 \mathrm{~V}$ then the system condition is in pattern $\mathrm{B}$.

Fig. 10 shows the experimental result when $V_{\mathrm{Lmin}}$ is $10 \mathrm{~V}$. It is confirmed that the PV simulator output voltage $V_{\mathrm{PV}}$ is correctly moved to the $V_{\mathrm{OP}}$. This is the bridge operation mode. $P_{\mathrm{PV}}$ increases to $40 \mathrm{~W}$ by this operation. After the bridge operation mode, the system mode transits the standby state mode. As a result, it is confirmed that the proposed system operates correctly.

Fig. 11 shows the experimental results when $V_{\text {Lmin }}$ is $20 \mathrm{~V}$. The input voltage $V_{\mathrm{PV}}$ of $15 \mathrm{~V}$ which is out of the operation range of PCS, is boosted to $30 \mathrm{~V}$ as the output voltage $V_{\mathrm{L}}$. $P_{\mathrm{PV}}$ increases to $40 \mathrm{~W}$ by this operation. It is confirmed that the PV simulator output voltage $V_{\mathrm{PV}}$ moves correctly to the $V_{\mathrm{OP}}$. As a result, it is made clear that the proposed system operates correctly.

\subsection{Main Experiment}

The effectiveness of proposed system can be evaluated by the amount of electric energy and operating voltage measured from input and output of the proposed system, comparing between the conventional system and the proposed system. As shown in Fig. 12, 2 PV panels are connected in series, and about 3/4 shadow is added to one cell from 9:00 am. The minimum operating voltage $V_{\mathrm{Lmin}}$ of PCS is set to $10 \mathrm{~V}$.

Fig. 13 shows $P-V$ characteristic with partial shadow. Furthermore, the optimum operating voltage $V_{\mathrm{OP}}$ of the PV panel is about $23 \mathrm{~V}$ after 9:00 am.

In this case, experimental condition is in pattern A. Table 2 shows the specifications of the PV panel.

Fig. 14 compares the acquired electrical power from the panels between the proposed system and conventional system. This figure shows an increase in the acquired electrical power when the proposed system is used. The generated electrical energy with conventional system is $318 \mathrm{Wh}$, the generated electrical energy with the proposed system shows an increase to $456 \mathrm{Wh}$. Therefore the generated electrical energy of daytime is 1.4 times increased as compared with the conventional system.

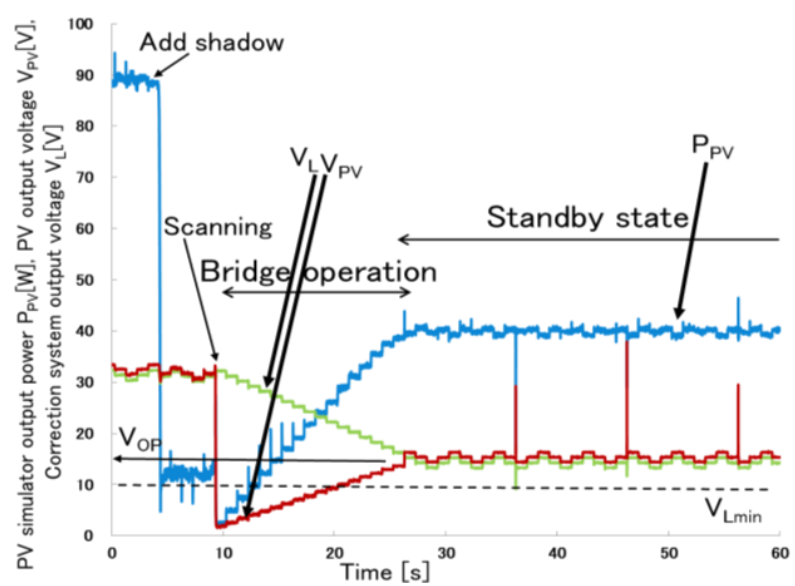

Fig. 10 When $V_{\text {Lmin }}$ is $10 \mathrm{~V}$.

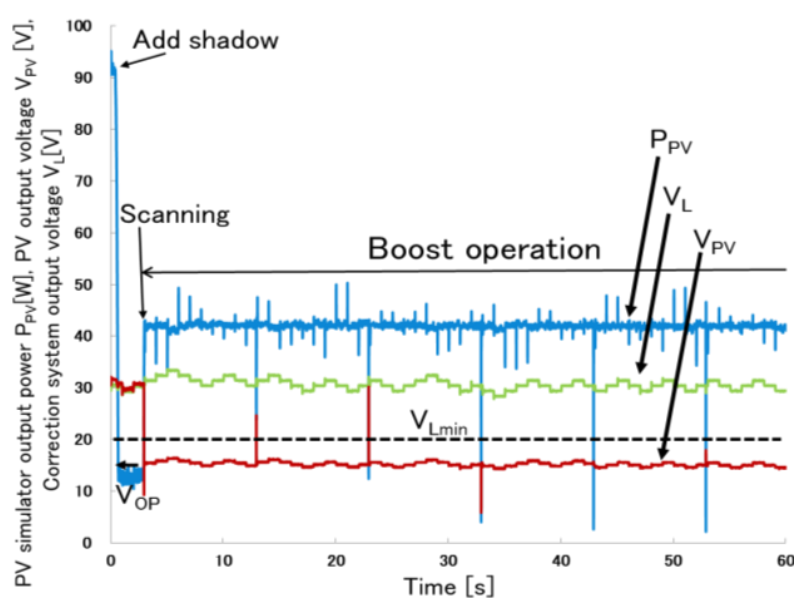

Fig. 11 When $V_{\mathrm{Lmin}}$ is $20 \mathrm{~V}$.

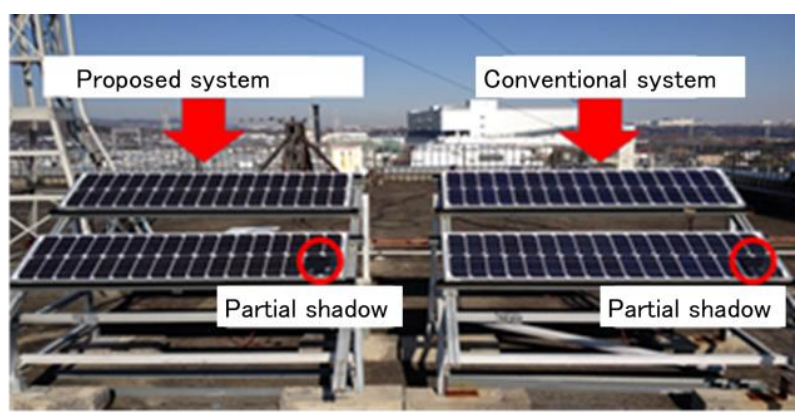

Fig. 12 Experimental landscape.

Table 2 Specifications of the PV module.

\begin{tabular}{ll}
\hline Maximum output $P_{\mathrm{MAX}}$ & $50 \mathrm{~W}$ \\
Open circuit voltage $V_{\mathrm{OC}}$ & $20.5 \mathrm{~V}$ \\
Short circuit current $I_{\mathrm{SC}}$ & $3.35 \mathrm{~A}$ \\
Voltage at maximum output $V_{\mathrm{OP}}$ & $16.4 \mathrm{~V}$ \\
Current at maximum output $I_{\mathrm{OP}}$ & $3.05 \mathrm{~A}$ \\
\hline
\end{tabular}




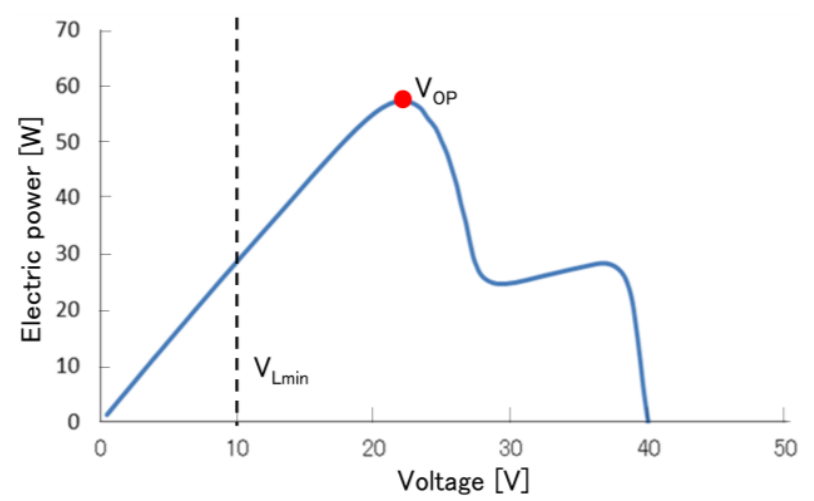

Fig. $13 P-V$ characteristic with partial shadow.

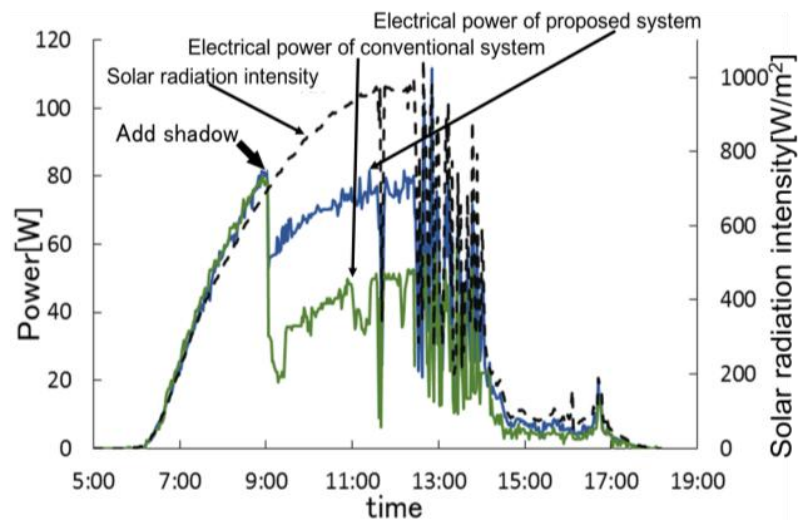

Fig. 14 PV output power characteristics.

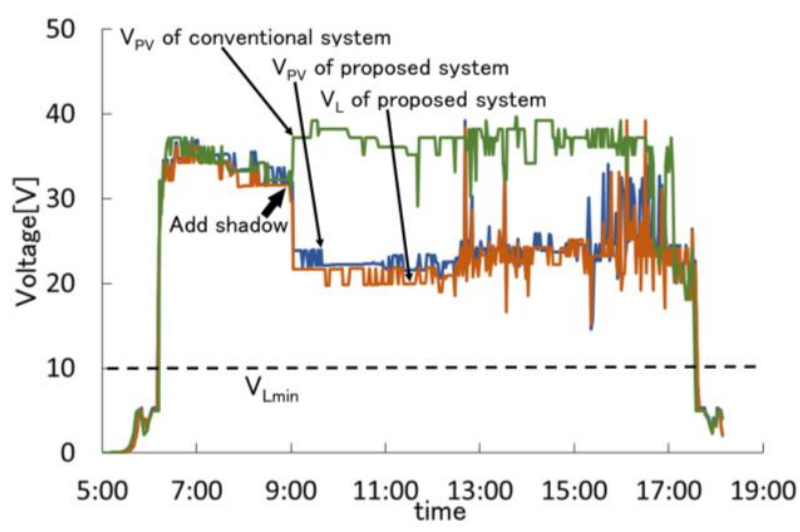

Fig. 15 The operating voltage comparison.

Furthermore, corrected time zone of the proposed system is from 9:00 am to 5:00 pm. In this time zone, the generated electrical energy with conventional system is $198 \mathrm{Wh}$, the generated electrical energy with the proposed system is $341 \mathrm{Wh}$. Therefore the generated electrical energy is 1.7 times increased by using the proposed system from 9:00 am to 5:00 pm.

Fig. 15 compares the proposed system operating voltage with conventional system operating voltage
$V_{\mathrm{PV}}$. In the conventional system, it is confirmed that the partial shadow is added, the operating point voltage $V_{\mathrm{PV}}$ does not go to $V_{\mathrm{OP}}$. However, in the proposed system, the operating point voltage $V_{\mathrm{PV}}$ is corrected to $V_{\mathrm{OP}}$ after the shadow addition. As a result, it is confirmed that the PV panels operate at the maximum power point by using the proposed system.

\section{Conclusions}

From these experimental results, it is confirmed that the proposed system operates correctly and the maximum power point is obtained by the PCS. In addition, it is clarified that by using this system when there is a partial shadow, the amount of generated electric energy under the present experimental conditions increases approximately 1.4 times, as compared with the conventional system.

\section{References}

[1] Subudhi, B., and Raseswari, P. 2013. "A Comparative Study on Maximum Power Point Tracking Techniques for Photovoltaic Power Systems." IEEE Transactions on Sustainable Energy 4 (1): 88-98.

[2] Hua, C., Lin, J., and Shen, C. 1998. "Implementation of a DSP-Controlled Photovoltaic System with Peak Power Tracking." IEEE Trans. Lnd. Electron 45 (1): 99-107.

[3] Koutroulis, E., Kalaitzakis, K., and Voulgaris, N. C. 2001. "Development of a Microcontoroller-Based Photovoltaic Maximum Power Point Tracking Control System." IEEE Trans. Power Electron 16 (1): 46-54.

[4] Femia, N., Petrone, G., Spagnuolo, G., and Vitelli, M. 2005. "Optimization of Perturb and Observe Maximum Power Point Tracking Method." IEEE Trans. Power Electronics 20 (4): 963-73.

[5] Itako, K., and Mori, T. 2006. "A New MPPT Control Method for PV Generation Systems." In Proceedings of the International Conference on Electrical Engineering 2006 (CD-ROM).

[6] Itako, K., and Mori, T. 2007. "A Single Sensor Type MPPT Control Method for PV Generation Systems." In Proceedings of the 12th European Conference on Power Electronics and Applications 2007 (CD-ROM).

[7] Itako, K. 2012. "A Detecting Interval Control in MPPT Control with I-V Characteristics Scanning for a PV Generation System." In Proceedings of the International Conference on Electrical Engineering 2012 (CD-ROM).

[8] Itako, K. 2013. "New I-V Characteristics Scan-Type 
MPPT Control Method for PV Generation System." Journal of Technology Innovations in Renewable Energy (USA) 1 (2): 87-91.

[9] Itako, K. 2013. "Power Conditioning System with New MPPT Control for a Grid-Connected PV Power Generation System." In Proceedings of PCIM Europe 2013 (CD-ROM).

[10] Itako, K., Daidouji, S., and Mori, T. 2010. "Characteristics in Partial Shadow of MPPT Control With I-V Characteristics Scanning." In Proceedings of the Renewable Energy 2010 (CD-ROM).

[11] Itako, K., Togashi, Y., Zama R., and Matsuura, Y. 2010. "Development of an MPPT Operating Point Correction
Unit for Improving Efficiency in a Photovoltaic System.” In Proceedings of the Renewable Energy 2010 (CD-ROM).

[12] Itako, K., Ochiai, M., Iiduka, N., and Hossam, B. 2016. "Study on New MPPT Operating Point Correction Unit for Improving Efficiency of the PV Generation System" In Proceedings of the International Conference on Electrical Engineering 2016 (CD-ROM).

[13] Itako, K., Ochiai, M., Iiduka, N., and Hossam, B. 2016. "Effect of New Operation Point Correction Unit for a PV Generation Systemwith Different Directional PV Panels." In Proceedings of the IEEE International Conference on Power and Renewable Energy 2016 (CD-ROM). 